Preprint solv-int/9801012

\title{
Dynamical boundary conditions for integrable lattices.
}

\author{
A.V. Tsiganov \\ Department of Mathematical and Computational Physics, Institute of Physics, \\ St.Petersburg University, 198 904, St.Petersburg, Russia
}

\begin{abstract}
Some special solutions to the reflection equation are considered. These boundary matrices are defined on the common quantum space with the other operators in the chain. The relations with the Drinfeld twist are discussed.
\end{abstract}

PACS: 02.90.+p, 03.20.+i,03.65.Fd, 11.30.-j 


\section{On a covariance property of the reflection equation}

Let the operator-valued function $R(u): \mathbb{C} \rightarrow \operatorname{Aut}(V \otimes V)$ be a solution to the quantum Yang-Baxter equation [1] in a finite-dimensional linear space $V$. Let us connect with a given matrix $R(u)$ two associative algebras $\mathcal{T}_{R}$ and $\mathcal{U}_{R}$ generated by noncommutative entries of the square matrices $T(u)$ and $K(u)$ satisfying the fundamental commutator relation [1] 2

$$
R_{12}(u-v) \stackrel{1}{T}(u) \stackrel{2}{T}(v)=\stackrel{2}{T}(v) \stackrel{1}{T}(u) R_{12}(u-v),
$$

or the reflection equation [3, 何

$$
R_{12}(u-v) \stackrel{1}{K}(u) R_{21}(u+v) \stackrel{2}{K}(v)=\stackrel{2}{K}(v) R_{12}(u+v) \stackrel{1}{K}(u) R_{21}(u-v) .
$$

Here $\stackrel{1}{X} \equiv X \otimes i d_{V_{2}}, \stackrel{2}{X} \equiv i d_{V_{1}} \otimes X$ for any matrix $X \in \operatorname{End}(V)$. As usual, $R_{i j}(u) \in \operatorname{End}\left(V_{i} \otimes V_{j}\right), V_{j} \equiv V$ and $R_{j i}(u)=P R_{i j}(u) P$, with $P$ as the permutation operator in the tensor product of the two spaces $V_{i} \otimes V_{j}$ [1, 2].

In this paper we discuss solutions to the reflection equation (1.2), i.e. consider various representations of the algebra $\mathcal{U}_{R}$ 泪. There is plenty of known representations [3, 田, 司, 6]. So, the covariance property of the reflection equation (1.2) [4, 7] may be used to construct new solutions starting from known ones. Namely, it was pointed out in 4 that

Proposition 1 Let the matrices $T(u)$ and $K(u)$ satisfy the relations (1.1) and (1.8) with the same $R$-matrix $R(u)$, then the Sklyanin monodromy matrix

$$
K^{\prime}(u)=T(u) K(u) T^{-1}(-u),
$$

solves the reflection equation 1.8 ) if

$$
\stackrel{1}{T}(u) \stackrel{2}{K}(v) \stackrel{2}{K}(v) \stackrel{1}{T}(u) .
$$

The proof follows easily by the substitution $K^{\prime}(u)$ into the reflection equation (1.2) and by using few times different forms of the fundamental relation (1.1) e.g.

$$
\stackrel{2}{T}^{-1}(-v) R_{12}(u+v) \stackrel{1}{T}(u)=\stackrel{1}{T}(u) R_{12}(u+v) \stackrel{2}{T^{-1}}(-v) .
$$

The main condition (1.4) holds if $T(u)$ and $K(u)$ are some representations of the algebras $\mathcal{T}_{R}$ and $\mathcal{U}_{R}$ in the different spaces $\mathcal{H}_{1} \otimes V$ and $\mathcal{H}_{2} \otimes V$ respectively, whence entries of $T(u)$ and $K(u)$ are mutually commute. Here $\mathcal{H}_{1}$ and $\mathcal{H}_{2}$ are the distinct quantum spaces and $V$ means a common auxiliary space [1, 2].

In this paper the Sklyanin monodromy matrix $K^{\prime}(1.3)$ is constructed from the known representations $T(u)$ and $K(u)$ of the algebras $\mathcal{T}_{R}$ and $\mathcal{U}_{R}$ defined on the common quantum space $\mathcal{H}$, i.e. using matrices with the non-ultralocal commutation relations [8]. In that case initial matrix $K(u)$ will be termed the dynamical boundary matrix.

Let us preserve construction of the Sklyanin representation $K^{\prime}(1.3)$ and refuse on the covariance property of the initial reflection equation (1.2).

Proposition 2 Let $T(u)$ satisfies the fundamental commutator relation (1.1). If it is intertwined with dynamical boundary matrix $K(u)$ by initial matrix $R(u)$ and by some new matrix $S(u, v)$

$$
\stackrel{1}{K}(u) \stackrel{2}{T}(v) R_{12}(u+v) \stackrel{1}{T}-1(-u) \stackrel{2}{K}(v)=\stackrel{2}{T}(v) \stackrel{1}{K}(u) S_{21}(u, v) \stackrel{2}{K}(v) \stackrel{1}{T}{ }^{-1}(-u),
$$

then the Sklyanin monodromy matrix

$$
K^{\prime}(u)=T(u) K(u) T^{-1}(-u),
$$

solves initial reflection equation (1.9) provided that the dynamical boundary matrix $K(u)$ satisfies the generalized reflection equation

$$
R_{12}(u-v) \stackrel{1}{K}(u) S_{21}(u, v) \stackrel{2}{K}(v)=\stackrel{2}{K}(v) S_{12}(u, v) \stackrel{1}{K}(u) R_{21}(u-v) .
$$


While $S(u, v)$ is an arbitrary matrix and the proof consists in direct verification of the reflection equation (1.2) by using relations (1.1, 1.6, 1.7).

For a given matrix $T(u)$ we can try to find dynamical boundary matrix $K(u)$ together with the matrix $S(u, v)$ from the equation (11.6), then we have to check the generalized reflection equation (1.7) intertwining both these matrices with the $R$-matrix.

Particular solutions to the equation (1.6) may be obtained from the following system of equations

$$
\begin{aligned}
\stackrel{1}{K}(u) \stackrel{2}{T}(v) & =\stackrel{2}{T}(v) \stackrel{1}{K}(u) F(u, v), \\
\stackrel{1}{T^{-1}}(-u) \stackrel{2}{K}(v) & =G(u, v) \stackrel{2}{K}(v) \stackrel{1}{T}-1(-u),
\end{aligned}
$$

with the two unknown matrices $F(u, v)$ and $G(u, v)$. In that case dynamical boundary matrix $K(u)$ has to be solution to generalized reflection equation (1.7) with the matrix

$$
S(u, v)=F(u, v) R(u+v) G(u, v) .
$$

Equations (1.8) have the form of the exchange algebras [9]. Obviously, other form of these algebras are

$$
\begin{aligned}
& F(u, v)=\stackrel{1}{K^{-1}}(u) \stackrel{2}{T^{-1}}(v) \stackrel{1}{K}(u) \stackrel{2}{T}(v) \\
& G(u, v)=\stackrel{1}{=} T^{-1}(-u) \stackrel{2}{K}(v) \stackrel{1}{T}(-u) \stackrel{2}{K^{-1}}(v) .
\end{aligned}
$$

Let the dynamical boundary matrix $K(u)$ has the following property

$$
K(u) K(-u)=\phi(u) I,
$$

where $\phi(u)$ means some scalar function. In that case matrix $S(u, v)(1.9)$ is the Drinfeld twist 10] of the matrix $R(u+v)$

$$
S(u, v)=F(u, v) R(u+v) F_{21}^{-1}(-v,-u), \quad F_{21}(u, v)=P F(u, v) P,
$$

if the universal twist element $F(u, v)$ has an appropriate properties 10$]$. Note, a twist transformation (1.11) of the $R$-matrix related to the braid group $\check{R}=P R$

$$
\check{S}(u, v)=P S(u, v)=F_{21}(u, v) \check{R} F_{21}^{-1}(-v,-u),
$$

looks just like a Sklyanin formulae (1.3), in contrast with the usual similarity transformation in the quantum group theory.

Recall, for the integrable lattice models matrix $T(u)$ is constructed as an ordered product

$$
T(u)=L_{n}(u) L_{n-1}(u) \cdots L_{1}(u),
$$

of $n$ independent $L$-operators having some simple dependence on spectral parameter $u$. Matrices $L_{k}(u)$ in the chain (1.12) are operators which act on the different local spaces $\mathcal{H}_{k} \otimes V$, now we use the notation $\mathcal{H}_{k}$ for a local quantum space assigned to the site $k$ in the lattice and $V \simeq \mathbb{C}^{n}$ is a common auxiliary space [1, 2].

Let $K(u)$ be representation of the reflection equation algebra $\mathcal{U}_{R}$ in the space $\mathcal{H}_{-} \otimes V$. Then, the Sklyanin monodromy matrix (1.3) 田, 司, 6]

$$
K_{-}(u)=L_{n}(u) L_{n-1}(u) \cdots L_{1}(u) K(u) L_{1}^{-1}(-u) L_{2}^{-1}(-u) \cdots L_{n}^{-1}(-u)
$$

describes a lattice model with boundary conditions corresponding to the matrix $K(u)$. As usual, transfer matrix is given by

$$
\tau(u)=\operatorname{tr} K_{+}(u) T(u) K(u) T^{-1}(-u)=\operatorname{tr} K_{+}(u) K_{-}(u) .
$$


Here an extra boundary $K$-matrix $K_{+}$is some solution of a "conjugated" reflection equation [4] on the quantum space $\mathcal{H}_{+}$defined in such way to guarantee the commutativity $[\tau(u), \tau(v)]=0$. This transfer matrix gives rise to the hamiltonian and other integrals of motion for a quantum system with the space of states $\mathcal{H}=\mathcal{H}_{+} \otimes \mathcal{H}_{n} \otimes \mathcal{H}_{n-1} \cdots \mathcal{H}_{1} \otimes \mathcal{H}_{-}$.

Looking for dynamical boundary matrices $K(u)$ we can start with a single operator $L(u)$ in the chain (1.13). In addition, we can begin either with the generalized reflection equation (1.7) by using known twists $S(u, v)$ [10, 16, 12], or with the exchange algebras (1.8) by assuming some ansatz for the boundary matrix $K(u)$.

\section{The Toda lattices}

As an example, let us consider the following $L$-operator

$$
L(u)=\left(\begin{array}{cc}
u-p & -\exp (q) \\
\exp (-q) & 0
\end{array}\right), \quad[p, q]=-i \eta,
$$

where $(p, q)$ be a pair of canonical conjugated variables. This $L$-operator is intertwined (1.1) by the rational Yang $R$-matrix

$$
R(u)=u I-i \eta P, \quad P=\left(\begin{array}{cccc}
1 & 0 & 0 & 0 \\
0 & 0 & 1 & 0 \\
0 & 1 & 0 & 0 \\
0 & 0 & 0 & 1
\end{array}\right)
$$

where $P$ be permutation operator in $\mathbb{C}^{2} \otimes \mathbb{C}^{2}$ and

$$
R(u)=\left(\begin{array}{cccc}
u-i \eta & 0 & 0 & 0 \\
0 & u & -i \eta & 0 \\
0 & -i \eta & u & 0 \\
0 & 0 & 0 & u-i \eta
\end{array}\right)=\left(\begin{array}{cccc}
f & 0 & 0 & 0 \\
0 & g & h & 0 \\
0 & h & g & 0 \\
0 & 0 & 0 & f
\end{array}\right)(u),
$$

In that case the monodromy matrix $T(u)(1.12)$ describes the Toda lattices associated with the root system $\mathcal{A}_{n}$ [18]. The corresponding hamiltonian reads as

$$
H_{A}=\sum_{j=1}^{n} \frac{1}{2} p_{j}^{2}+\sum_{j=1}^{n-1} \exp \left(q_{j+1}-q_{j}\right)
$$

The set of the operators $\left\{L\left(q_{j}, p_{j}, u\right)\right\}_{j=1}^{n}$ (2.1) defines the monodromy matrix $T(u)$ (1.12), which is a spin- $1 / 2$ representation of the Yangian $Y(\operatorname{sl}(2))$ in $V=\mathbb{C}^{2}$. So, the inversion $T^{i}(u)=T^{-1}(-u)$ is equal to [4]

$$
T^{i}(u)=\sigma_{2} T^{t}(-u-i \eta) \sigma_{2} / \Delta\left\{T\left(-u-\frac{i \eta}{2}\right)\right\} .
$$

where $t$ means a matrix transposition, $\sigma_{2}$ is the Pauli matrix and $\Delta\{T(u)\}$ is a quantum determinant of $T(u)$. According to general recipe $\llbracket$, it allows us to work with the algebra $\widetilde{\mathcal{U}}_{R}$ instead of the $\mathcal{U}_{R}$. The new algebra $\widetilde{\mathcal{U}}_{R}$ has the following Sklyanin representation

$$
K_{-}=T(u) K\left(u-\frac{i \eta}{2}\right) \sigma_{2} T^{t}(-u) \sigma_{2} .
$$

Let us begin with the known scalar solution of the reflection equation (1.2)

$$
K_{c}(u)=\left(\begin{array}{cc}
a & b \\
c & d
\end{array}\right)(u) \equiv\left(\begin{array}{cc}
\alpha u+\delta & \beta u \\
\gamma u & -\alpha u+\delta
\end{array}\right)
$$

where $\alpha, \beta, \gamma$ and $\delta$ are complex numbers. By using the monodromy matrix $T(u)(1.12)$, the usual covariance property and solution $K_{c}(u)$ 2.7) one can get the monodromy matrix $K_{-}(2.6)$, which 
describes the Toda lattices associated with the root system $\mathcal{A}_{n}$ by $\alpha=\delta=\gamma=0$ (2.4) and with the root system $\mathcal{B C}_{n}$ by $\beta=1$ [ [⿴囗十

$$
H_{B C}=H_{A}-\frac{\gamma}{2} \exp \left(2 q_{1}\right)+\left(\delta-\alpha p_{1}\right) \exp \left(q_{1}\right) .
$$

Transfer matrix (1.14) with the "conjugated" to $K_{c}$ (2.7) matrix $K_{+}$allows to describe another Toda lattices associated to the several affine root systems [11, 4, 5].

Looking for dynamical boundary matrix $K(u)$ let us begin with the single operator $L(u)$ (2.1) in the chain and introduce the following ansatz for the dynamical boundary matrix

$$
K(u)=\left(\begin{array}{ll}
a & b \\
c & d
\end{array}\right)(q, u)
$$

where $a, b, c$ and $d$ are functions of spectral parameter $u$ and one dynamical variable $q$ only. This matrix $K(u)(2.9)$ depends on the half dynamical variables and, therefore, may be constructed from the scalar solutions to generalized reflection equation.

Inserting ansatz $K(q, u)(2.9)$ into the dynamical exchange algebras (1.8) and then into the general dynamical equation (1.6) we get two nontrivial upper $c(q, u)=0$ and lower $b(q, u)=0$ triangular matrices. In both these solutions diagonal entries $a(u)$ and $d(u)$ are independent on the dynamical variable $q$.

Note, triangular boundary matrices with the property $(1.10)$ are obtained from the dynamical equations $(1.6-1.8)$ by using the special ansatz (2.9). Only than, due to the special structure of the boundary matrices, we can see that the transformation (1.9) of the Yang solution $R(u)(2.3)$ is just the Drinfeld twist (1.11) depending on the spectral parameters [10] only. Moreover, these twists are closely connected to the twists of the underlying Lie algebra $s l(2)$.

\subsection{Lower triangular dynamical matrix.}

Inserting the lower triangular matrix

$$
K_{d}=\left(\begin{array}{cc}
a(u) & 0 \\
c(q, u) & d(u)
\end{array}\right),
$$

into the system (1.8) one gets two dynamical equations

$$
[p, c(q, u)]=-z(q, u) d(u) \exp (q), \quad[p, c(q, v)]=z^{\prime}(q, v) a(v) \exp (q),
$$

related with the following matrices

$$
F=\left(\begin{array}{cccc}
1 & 0 & 0 & 0 \\
0 & 1 & 0 & 0 \\
0 & 0 & 1 & 0 \\
z(q, u) & 0 & 0 & 1
\end{array}\right), \quad G=\left(\begin{array}{cccc}
1 & 0 & 0 & 0 \\
0 & 1 & 0 & 0 \\
0 & 0 & 1 & 0 \\
z^{\prime}(q, v) & 0 & 0 & 1
\end{array}\right)
$$

Here $z(q, u)$ and $z^{\prime}(q, v)$ are functions of the spectral parameters and of the dynamical variable $q$. By using generators $\mathbf{h}, \mathbf{e}, \mathbf{f}$ of the underlying Lie algebra $\operatorname{sl}(2)$

$$
[\mathbf{h}, \mathbf{e}]=2 \mathbf{e}, \quad[\mathbf{h}, \mathbf{f}]=-2 \mathbf{f}, \quad[\mathbf{e}, \mathbf{f}]=\mathbf{h},
$$

let us introduce an appropriate element $\mathcal{F} \in U(s l(2)) \otimes U(s l(2))$

$$
\mathcal{F}_{z}=\exp (z \cdot \mathbf{f} \otimes \mathbf{f}), \quad z \in \mathbb{C} .
$$

belonging to a tensor product of the corresponding universal enveloping algebras $U(s l(2))$ [12]. In the fundamental spin- $1 / 2$ representation $\rho_{\frac{1}{2}}$ we have

$$
F=\left(\rho_{\frac{1}{2}} \otimes \rho_{\frac{1}{2}}\right) \mathcal{F}_{z}, \quad G=\left(\rho_{\frac{1}{2}} \otimes \rho_{\frac{1}{2}}\right) \mathcal{F}_{z^{\prime}}
$$


However, now $z$ and $z^{\prime}$ are the proper functions of both spectral parameters. The associated twist (1.9)

$$
\begin{aligned}
S(u, v) & =F R(u+v) F_{21}^{-1}=I_{z z^{\prime}}[(u+v) I-i \eta P] \\
I_{z z^{\prime}} & =\exp \left(\left(z+z^{\prime}\right) \cdot \mathbf{f} \otimes \mathbf{f}\right)
\end{aligned}
$$

is equal to

$$
S(u, v)=F R(u+v) G=\left(\begin{array}{cccc}
f & 0 & 0 & 0 \\
0 & g & h & 0 \\
0 & h & g & 0 \\
{\left[z(q, u)+z^{\prime}(q, v)\right]} & 0 & 0 & f
\end{array}\right)(u, v)
$$

Next, the only possible solution to the dynamical equations (2.11) is given by

$$
K_{d}=\left(\begin{array}{cc}
u & 0 \\
u \gamma(q) & -u
\end{array}\right)
$$

Here entry $\gamma(q)$ is defined by

$$
[p, \gamma(q)]=z(q) \exp (q)
$$

with an arbitrary function $z(q)$ of dynamical variable $q$ and $z(q)=-z^{\prime}(q)$, whence $S(u, v)=R(u+v)$.

Next, we have to solve the general dynamical equation (1.6). Let the matrix $S(u, v)$ is equal to

$$
S(u, v)=\left(\begin{array}{cccc}
f & 0 & 0 & 0 \\
0 & g & h & 0 \\
0 & h & g & 0 \\
z(q, u, v) & 0 & 0 & f
\end{array}\right)(u, v)
$$

where $z(q ; u, v)$ is an arbitrary entry. Substituting this matrix and the matrix $K_{d}(2.10)$ into $(1.6)$ one gets the following dynamical equation

$$
a(v)[p, c(q, u)]+d(u)[p, c(q, u)]=z(q ; u, v) \frac{a(v) d(u)}{u+v-i \eta} \exp (q),
$$

which by $c(q, u)=\gamma(q) u$ reads as

$$
[p, \gamma(q)]=z(q ; u, v) \frac{a(v) d(u)}{(u a(v)+v d(u))(u+v-i \eta)} \exp (q)
$$

Solving the generalized reflection equation (1.7) with this matrix $S(u, v)$ one gets the same possible nontrivial solution $K_{d}(2.10)$ only.

To construct the transfer matrix $\tau(u)(1.14)$ let us substitute $q=q_{1}$ in the matrix $K_{d}(q, u)$ and $q=-q_{n}$ in the "conjugated" matrix $K_{+}(q, u)=K_{d}^{t}\left(-q_{n}, u\right)$. Thus dynamical boundary matrices $K_{d}$ and $K_{d+}$ have the common quantum spaces with the first $L_{1}$ and with the last $L_{n}$ operators in the lattice. This generating polynomial

$$
\begin{array}{r}
\tau(u)=\operatorname{tr}\left(K_{d}^{t}\left(-q_{n}, u+\frac{i \eta}{2}\right) L_{n}\left(q_{n}, u\right) \ldots L_{1}\left(q_{1}, u\right) \times\right. \\
\left.\times K_{d}\left(q_{1}, u-\frac{i \eta}{2}\right) \sigma_{2} L_{1}^{t}\left(q_{1},-u\right) \ldots L_{n}^{t}\left(q_{n},-u\right) \sigma_{2}\right)
\end{array}
$$

has the form

$$
\tau(u)=H_{1} u^{2 n}+H_{2} u^{2 n-2}+\ldots
$$

and it gives rise the commutative family of $n$ functionally independent integrals of motion. If the entry $z(u, v, q)$ is independent on dynamical variable $q$ then solution to equations (2.13) and (2.15) is equal to

$$
\gamma(q)=\gamma \exp (q)+\beta
$$


whereas first integral $H_{1}$ has the following factorable form

$$
H_{1}=J_{1} \cdot J_{n}=\left(2 p_{1}+\gamma_{-} e^{2 q_{1}}+\beta_{-} e^{q 1}\right) e^{q_{1}} \cdot e^{-q_{n}}\left(-2 p_{n}+\gamma_{+} e^{-2 q_{n}}+\beta_{+} e^{-q n}\right) .
$$

Here $\left(\gamma_{-}, \beta_{-}\right)$and $\left(\gamma_{+}, \beta_{+}\right)$are the free parameters associated to the boundary matrices $K_{d}\left(q_{1}, u\right)$ and $K_{d}^{t}\left(-q_{n}, u\right)$, respectively.

This integrable system may be considered as the constrained hamiltonian system either with one constraint $H_{1}=$ const, or with two constraints

$$
q_{1}=\text { const }_{1}, q_{n}=\text { const }_{n}, \quad \text { or } \quad J_{1}=\text { const }_{1}, J_{n}=\text { const }_{n} .
$$

\subsection{Upper triangular dynamical matrix.}

Inserting upper triangular matrix

$$
K_{D}=\left(\begin{array}{cc}
a(u) & b(q, u) \\
0 & d(u)
\end{array}\right)
$$

into the system (1.8) one gets two dynamical equations

$$
[p, b(q, u)]=-w(q, u) a(u) \exp (q), \quad[p, b(q, v)]=-w^{\prime}(q, v) d(v) \exp (q),
$$

related with the following matrices

$$
F=\left(\begin{array}{cccc}
1 & 0 & 0 & 0 \\
0 & 1 & w(q, u) & 0 \\
0 & 0 & 1 & 0 \\
0 & 0 & 0 & 1
\end{array}\right), \quad G=\left(\begin{array}{cccc}
1 & 0 & 0 & 0 \\
0 & 1 & 0 & 0 \\
0 & w^{\prime}(q, v) & 1 & 0 \\
0 & 0 & 0 & 1
\end{array}\right)
$$

Here $w(q, u, v)$ and $w^{\prime}(q, u, v)$ are functions of spectral parameters and dynamical variable $q$.

In generators (2.12) the corresponding twist element $\mathcal{F} \in U(s l(2)) \otimes U(s l(2))$ is equal to

$$
\mathcal{F}_{w}=\exp (w \cdot \mathbf{f} \otimes \mathbf{e}), \quad w \in \mathbb{C}
$$

(see factorization of the universal $R$-matrix in 12, 16). In the fundamental spin- $1 / 2$ representation $\rho_{\frac{1}{2}}$ we have

$$
F=\left(\rho_{\frac{1}{2}} \otimes \rho_{\frac{1}{2}}\right) \mathcal{F}_{w}, \quad G=P\left(\rho_{\frac{1}{2}} \otimes \rho_{\frac{1}{2}}\right) \mathcal{F}_{w^{\prime}} P .
$$

However, now $w$ and $w^{\prime}$ are the corresponding to (2.17) functions of the spectral parameters. We can see, $G=F_{21}^{-1}$ up to change the twist parameters $w(q, u) \rightarrow-w^{\prime}(q, v)$. The associated twisted matrix $S(u, v)(1.9)$ is equal to

$$
S(u, v)=F_{12}(w)((u+v) I-i \eta P) F_{21}^{-1}\left(w^{\prime}\right)=J_{w w^{\prime}}-i \eta I_{w w^{\prime}} P,
$$

where

$$
\begin{aligned}
& J_{w w^{\prime}}=\left(\rho_{\frac{1}{2}} \otimes \rho_{\frac{1}{2}}\right) \exp \left(w \cdot \mathbf{f} \otimes \mathbf{e}+w^{\prime} \cdot \mathbf{e} \otimes \mathbf{f}\right), \\
& I_{w w^{\prime}}=\left(\rho_{\frac{1}{2}} \otimes \rho_{\frac{1}{2}}\right) \exp \left(\left(w+w^{\prime}\right) \cdot \mathbf{f} \otimes \mathbf{e}\right) .
\end{aligned}
$$

In fact, matrix $S(u, v)$ has the following form

$$
S(u, v)=\left(\begin{array}{cccc}
f & 0 & 0 & 0 \\
0 & g+h\left(w+w^{\prime}\right)+w w^{\prime} g & h+w g & 0 \\
0 & h+w^{\prime} g & g & 0 \\
0 & 0 & 0 & f
\end{array}\right)(u, v),
$$

where $f, g$ and $h$ are entries of the initial $R$-matrix (2.3).

For a given $S$-matrix (2.19) we shall not solve dynamical equations (2.17) and generalized reflection equation (1.7) in generic. Here we restrict ourselves to those particular solutions which are related to interesting physical systems only. 
At first, let us introduce upper triangular matrix $K_{D}$ with the following entries

$$
a(u)=d(u)=u, \quad b(q, u)=\beta \exp (q)+\gamma,
$$

One immediately gets

$$
w(u)=i \eta \beta u^{-1}, \quad w^{\prime}(v)=i \eta \beta v^{-1},
$$

where parameters $w$ and $w^{\prime}$ of the twist are independent on the dynamical variable $q$. Thus, from the linear matrix-function $R(u)(2.3)$ we obtain the rational matrix $S(u, v)$, which has the upper and lower triangular residues at the points $u=0$ and $v=0$, respectively.

The second more complicated solution $K_{g D}$ is defined by

$$
a(u)=u^{2}+\alpha u+\delta, \quad d(u)=a(-u), \quad b(q, u)=(\beta \exp (q)+\gamma) u .
$$

For the both solutions

$$
K(u) K(-u)=\mp \phi(u) I,
$$

where function $\phi(u)$ is equal to the determinant of the matrices $K_{D}$ and $K_{g D}$, respectively.

To construct the transfer matrix $\tau(u)(1.14)$ let us substitute $q=q_{1}$ in the matrix $K_{D}(q, u)$ and $q=-q_{n}$ in the "conjugated" matrix $K_{+}(q, u)=K_{D}^{t}\left(-q_{n}, u\right)$. It means that dynamical boundary matrices $K_{D}$ and $K_{D+}$ have the common quantum spaces with the first $L_{1}$ and the last $L_{n}$ operators in the lattice. The generating polynomial $\tau(u)(1.14)$

$$
\begin{aligned}
\tau(u) & =\operatorname{tr}\left(K_{D}^{t}\left(-q_{n}, u+\frac{i \eta}{2}\right) L_{n}\left(q_{n}, u\right) \cdots L_{1}\left(q_{1}, u\right) \times\right. \\
& \left.\times K_{D}\left(q_{1}, u-\frac{i \eta}{2}\right) \sigma_{2} L_{1}^{t}\left(q_{1},-u\right) \cdots L_{n}^{t}\left(q_{n},-u\right) \sigma_{2}\right)
\end{aligned}
$$

has the form

$$
\tau(u)=H_{1} u^{2 n+2}+H_{2} u^{2 n}+\ldots,
$$

and it gives rise to the commutative family of $n+1$ functionally independent integrals of motion. The use of the second dynamical matrix $K_{g D}$ (2.21) leads to the similar results.

For the both solutions first integral $H_{1}$ into expansion (2.22) may be considered as the factorable constraint

$$
H_{1}=J_{1} \cdot J_{n}=\left(-2 e^{q_{1}}+\beta_{-} e^{q_{1}}+\gamma_{-}\right) \cdot\left(2 e^{-q_{n}}+\beta_{+} e^{-q n}+\gamma_{+}\right) .
$$

Here $\left(\gamma_{-}, \beta_{-}\right)$and $\left(\gamma_{+}, \beta_{+}\right)$are the free parameters associated to dynamical entries of the boundary matrices $K_{D}\left(q_{1}, u\right)$ and $K_{D+}=K_{D}^{t}\left(-q_{n}, u\right)$, respectively.

In contrast with the lower triangular solution this constraint is easy removed, if the parameters $w$ and $w^{\prime}$ of the twist are independent on the dynamical variable $q$. Namely, unless otherwise indicated, set

$$
\beta_{ \pm}=\mp 2
$$

such that $H_{1}=\gamma_{-} \gamma_{+}=$const and generating polynomial (2.22) gives rise to $n$ independent integrals of motion only.

After canonical transformation of the variables

$$
e^{q} \rightarrow 1-\operatorname{ch}(q),
$$

in the first $\mathcal{H}_{1}$ and in the last $\mathcal{H}_{n}$ local quantum spaces in the chain, the associated hamiltonians $H$ read as

$$
\begin{aligned}
H_{D} & =H_{A}+\exp \left(-q_{n-1}-q_{n}\right)+\exp \left(q_{1}+q_{2}\right) \\
H_{g D} & =H_{D}+\frac{\alpha_{1}}{\sinh ^{2} \frac{q_{1}}{2}}+\frac{\alpha_{2}}{\sinh ^{2} q_{1}}+\frac{\alpha_{3}}{\sinh ^{2} \frac{q_{n}}{2}}+\frac{\alpha_{4}}{\sinh ^{2} q_{n}} .
\end{aligned}
$$

Here four constants $\alpha_{j}$ are functions of the four initial constants $\alpha_{ \pm}$and $\delta_{ \pm}$in diagonal entries (2.21) of the boundary matrices $[5]$. 
Thus, dynamical boundary matrix $K_{D}(2.20)$ corresponds to the Toda lattices associated with the root system $\mathcal{D}_{n}$ [1]. Since, the single spin-1/2 representation $T(u)(1.12,2.1)$ of the Yangian $Y(\operatorname{sl}(2))$ may be used to construct the monodromy matrices for the Toda lattices associated to all the classical infinite series of the root systems. The second solution $K_{g D}(2.21)$ allows to add four extra parameters in the hamiltonian $H_{D}$ and it is known Inozemtsev's generalization of the Toda system [13].

Boundary matrices $K_{D}^{\prime}=L(u) K_{D} L^{-1}(-u)$ and $K_{g D}^{\prime}=L(u) K_{g D} L^{-1}(-u)$ were at first found in [5] starting from the known $2 n \times 2 n$ Lax matrices [13]. They are solutions to the usual reflection equation, which have the ultralocal commutation relations with other matrices in the chain. Note, we have to use the two different representations $T(u)(1.12)$ of the Yangian $Y(\operatorname{sl}(2))$ to describe Toda lattices associated with the $\mathcal{B C}_{n}$ and $\mathcal{D}_{n}$ root systems by using matrices $K_{c}$ and $K_{D}^{\prime}$, respectively. In the classical mechanics factorization $K_{D}^{\prime}=L(u) K_{D}(q, u) L^{-1}(-u)$ on the terms with non-ultralocal commutator relations has been applied to separation of variables in 15].

Two outer automorphisms of the space of infinite-dimensional representations of the Lie algebra $s l(2)$ be used to recover these boundary matrices $K_{D}^{\prime}$ and $K_{g D}^{\prime}$ in 14. It would be interesting to study interrelations among these automorphisms of $\operatorname{sl}(2)$ and the twists (1.11) of the usual rational $R$-matrix.

The relativistic Toda lattices associated to the $\mathcal{D}_{n}$ root systems 19 may by easy embedded in the proposed scheme as well. In that case the corresponding $R$-matrix is the known trigonometric solution to the Yang-Baxter equation [19] and the associated twist is connected to the twist of the algebra $s l_{q}(2)$.

\subsection{The Drinfeld twist and separation of variables method.}

We know that the representation theory of the Drinfeld twists may be very useful in the framework of the algebraic Bethe ansatz [16. According to [16], for the $X X X-\frac{1}{2}$ and $X X Z-\frac{1}{2}$ Heisenberg (inhomogeneous) quantum spin chains of finite length $n$ associated $\mathcal{F}$-matrices diagonalize the generating matrix of scalar products of quantum states of these models. They also diagonalize the diagonal (operator) entries of the quantum monodromy matrix.

Now we shortly discuss interrelations of the Drinfeld twists and the separation of variables method proposed by Sklyanin [17. For sake of brevity we shall work with the corresponding classical objects.

Starting from the known $2 n \times 2 n$ Lax matrices we can get solutions $K_{D}^{\prime}$ or $K_{g D}^{\prime}$ to the reflection equation (1.2) with ultralocal commutation relations. In the classical mechanics the associated $2 \times 2$ Lax matrix is equal to

$$
\begin{aligned}
\mathcal{L}^{\prime}(u) & =K_{D}^{\prime t}\left(-q_{n}, u\right) L_{n-1}\left(q_{n-1}, u\right) \cdots L_{2}\left(q_{2}, u\right) \times \\
& \times K_{D}^{\prime}\left(q_{1}, u\right) \sigma_{2} L_{2}^{t}\left(q_{2},-u\right) \cdots L_{n-1}^{t}\left(q_{n-1},-u\right) \sigma_{2} .
\end{aligned}
$$

Here all the local matrices $L_{j}(u), j=2, \ldots, n-1$ and boundary matrices $K_{D}^{\prime}\left(q_{1, n}, u\right)$ are defined on the different phase spaces such that $\left\{L_{j}^{1}(u), \stackrel{2}{K}_{D}^{\prime}\left(q_{1, n}, v\right)\right\}=0$.

In the Sklyanin approach [17] the use of this Lax matrix forces to apply the dynamical normalization of the corresponding Baker-Akhiezer vector-function $\Psi$ [15. Recall, the choice of the proper normalization $\vec{\alpha}[17$

$$
(\vec{a}, \Psi)=\sum_{j=1} \alpha_{j}(u) \Psi_{j}(u)=1
$$

allows us to fix special analytical properties of this meromorphic eigenfunction $\Psi$ of the Lax matrix $\mathcal{L}^{\prime}(u)$

$$
\mathcal{L}^{\prime}(u) \Psi=z \Psi .
$$

An appropriate normed vector-function $\Psi$ has to possess the necessary number of poles in involution and all the extra poles of $\Psi$ be constants 17]. However, one does not usually know the separating normalization in advance.

It is clear, using a similarity transformation for the Lax matrix $\mathcal{L}^{\prime}(u)$

$$
\mathcal{L}^{\prime}(u) \rightarrow \mathcal{L}(u)=V(u) \mathcal{L}^{\prime}(u) V^{-1}(u),
$$

the any normalization $\vec{a}$ may be turns into the simplest constant normalization vector 17$]$. So, in the classical mechanics the problem is to find an appropriate similarity transformation 2.26 ). 
In the quantum mechanics an action of the similarity transformations (2.26) may be transferred into the $R$-matrix level. If we permit to transform $R$-matrix in the special twists only, we essentially restrict the freedom related to similarity transformations (2.26). The twisted $R$-matrix enjoys most the properties of the usual $R$-matrix [10] and possible that it gains some new properties. As an example, admissible [10] or factorizing [16] twists may be used.

In this paper, starting from the known Yang solution $R(u)$ of the Yang-Baxter equation we introduce twist $S(u, v)$, which has another analytical properties. Next, solving the corresponding dynamical equations and the generalized reflection equation we get dynamical boundary matrices $K_{D}$ or $K_{g D}$. The associated $2 \times 2$ Lax matrix is equal to

$$
\begin{aligned}
\mathcal{L}(u) & =K_{D}^{t}\left(-q_{n}, u\right) L_{n}\left(q_{n}, u\right) \cdots L_{1}\left(q_{1}, u\right) \times \\
& \times K_{D}\left(q_{1}, u\right) \sigma_{2} L_{1}^{t}\left(q_{1},-u\right) \cdots L_{n}^{t}\left(q_{n},-u\right) \sigma_{2} .
\end{aligned}
$$

Here two local matrices $L_{1, n}(u)$ and boundary matrices $K_{D}\left(q_{1, n}, u\right)$ are defined on the common phase spaces and the corresponding Poisson brackets relations $\left\{L_{1, n}(u), \stackrel{2}{K}_{D}\left(q_{1, n} v\right)\right\} \neq 0$ may be derived from the quantum algebras (1.8).

Two Lax matrices $\mathcal{L}^{\prime}(u)(2.25)$ and $\mathcal{L}(u)(2.27)$ are related by the similarity transformation $(2.26)$. Now $V(u)$ is equal to the inversion matrix $V(u)=L_{n}^{i}\left(q_{n},-u\right)$ (2.5). This transformation leads to the appearance of the twist $S(u, v)(2.19)$ instead of the Yang solution $R(u+v)$ in the quantum algebraic relations.

Although matrices $K_{D}$ and $K_{g D}$ are solutions to the more complicated generalized reflection equation (1.7) and dynamical equations (1.6), nevertheless these matrices make possible to use the simplest constant normalization of the associated Baker-Akhiezer function [17] in the classical mechanics. Namely, for the Toda lattice associated to the $\mathcal{D}_{n}$ root system in the classical mechanics the Lax matrix $\mathcal{L}(u)$ (2.27) has the following matrix form

$$
\mathcal{L}(u)=\left(\begin{array}{cc}
A & B \\
C & D
\end{array}\right)(u)
$$

Choosing the simplest constant normalization $\vec{\alpha}=(1,0)$ of the associated Baker-Akhiezer function 17 the separation variables $\left\{x_{j}\right\}_{j=1}^{n}$ are defined as zeroes of the entry $B(u)$ 115

$$
B\left(u=x_{j}\right)=0,
$$

according to general recipe [17. It is easy to prove that they are real eigenvalues of the symmetric matrix defined by recursion, which has been proposed in [5. Other separation variables are sitting on the spectral curve of the Lax matrix (2.28) with the defined above variables $\left\{x_{j}\right\}_{j=1}^{n}$ [17, 15].

Thus, in the considered above example, the use of the Drinfeld twists in the quantum case leads to the suitable Lax representation and to the simplest separating normalization in the classical mechanics. We can see that the algebraic properties of the Lax matrix relate to the analytical properties of its eigenfunction. Of course, we have to study the suitable properties of this twist, which correspond to the separation of variables. Note, the constant normalization of the Baker-Akhiezer function allows to develop the quantum counterpart of the classical separation of variables method within the $R$-matrix approach [18] for the Toda lattices.

\section{Integrable tops closed to the Toda lattice.}

Let variables $l_{i}, g_{i}, i=1,2,3$ be generators of the Lie algebra $e(3)$ with commutator relations

$$
\begin{aligned}
& {\left[l_{i}, l_{j}\right]=-i \eta \mathbf{e}_{i j k} l_{k}, \quad\left[l_{i}, g_{j}\right]=-i \eta \mathbf{e}_{i j k} g_{k},} \\
& {\left[g_{i}, g_{j}\right]=0, \quad i, j=1,2,3,}
\end{aligned}
$$

and with the following Casimir operators

$$
J_{1}=(g, g), \quad J_{2}=(l, g) .
$$


Let us introduce quantum operator $T(u)$ for the Neumann's system

$$
T(u)=\left(\begin{array}{cc}
u^{2}-2 l_{3} u-l_{1}^{2}-l_{2}^{2}-\frac{1}{4} & i\left(g_{+} u-\frac{1}{2}\left\{g_{3}, l_{+}\right\}\right) \\
i\left(g_{-} u-\frac{1}{2}\left\{g_{3}, l_{-}\right\}\right) & g_{3}^{2}
\end{array}\right),
$$

here braces $\{$,$\} mean an anticommutator. Operator T(u)$ (3.1) at the level $J_{2}=(l, g)=0$ obeys the fundamental commutator relations (1.1) with the rational $R$-matrix (2.3) and closely related to the Toda system 20.

The use of the usual covariance property (1.3), operator $T(u)(3.1$ ) and the constant boundary matrices $K_{c}(2.7)$ allows to describe the quantum Kowalewski-Chaplygin-Goryachev top [20].

Let us consider the known constant solution (2.20) or (2.21) to the generalized reflection equation (1.7) with the considered above twisted matrix $S(u, v)(2.19)$. By using this solution we may introduce another solution of the same equation (1.7), which is the function defined on the Abel subalgebra of the $e(3)$. Thus, we can get dynamical boundary matrices on the common quantum space with operator $T(u)$ 3.1

$$
\begin{gathered}
K_{-}=\left(\begin{array}{cc}
u^{2}+\alpha_{-} u+\delta_{-} & i u\left(\beta_{-} g_{+}+\gamma_{-}\right) \\
0 & u^{2}-\alpha_{-} u+\delta_{-}
\end{array}\right), \\
K_{+}=\left(\begin{array}{cc}
u^{2}+\alpha_{+} u+\delta_{+} & 0 \\
i u\left(\beta_{+} g_{-}+\gamma_{+}\right) & u^{2}-\alpha_{+} u+\delta_{+}
\end{array}\right),
\end{gathered}
$$

such that

$$
\stackrel{1}{K_{+}} \stackrel{2}{K}_{-}=\stackrel{2}{K_{-}} \stackrel{1}{K_{+}}
$$

If we set

$$
\beta_{ \pm}=-2,
$$

then the generating polynomial (1.14) is equal to

$$
\tau(u)=\operatorname{tr}\left[K_{+} T(u) K_{-} \sigma_{2} T^{t}(-u) \sigma_{2}\right]=u^{6}\left(2 J_{1}-\gamma_{+} \gamma_{-}\right)+u^{4} J_{3}+u^{2} J_{4} .
$$

Two independent integrals of motion $J_{3}$ and $J_{4}$ are mutually commute at the level $J_{2}=(l, g)=0$. Here we present the corresponding hamiltonian $J_{3}$ in the classical mechanics only

$$
\begin{aligned}
J_{3} & =l_{+} l_{-}\left(g_{+} \gamma_{+}-\gamma_{+} \gamma_{-}+g_{-} \gamma_{-}\right)-2 l_{3}^{2}\left(2 g_{-}-\gamma_{+}\right)\left(2 g_{+}-\gamma_{-}\right) \\
& +2 l_{3}\left[2 J_{1}\left(\alpha_{+}+\alpha_{-}\right)-\left(\alpha_{-} \gamma_{+} g_{+}+\alpha_{+} \gamma_{-} g_{-}\right)\right] \\
& +l_{3} g_{3}\left[2 l_{-}\left(2 g_{+}-\gamma_{-}\right)+2 l_{+}\left(2 g_{-}-\gamma_{+}\right)-J_{3}\left(\alpha_{+}+\alpha_{-}\right)\right] \\
& +g_{3}\left(\gamma_{+} \alpha_{-} l_{+}+\alpha_{+} \gamma_{-} l_{-}\right)+\delta_{+} \gamma_{-} g_{-}+\delta_{-} \gamma_{+} g_{+} .
\end{aligned}
$$

By analogy with (2.23), the use of automorphisms of the Lie algebra $e(3)$ might allow to rewrite this hamiltonian in the more physical form. For us it is more important that all three matrices in the chain are defined on the single common quantum space.

\section{Conclusions}

We discuss dynamical boundary matrices defined on the common quantum space with other operators in the chain. These matrices are solutions to generalized reflection equation and dynamical exchange equations. These equations include usual $R$-matrix and its Drinfeld twist depending on the spectral parameters. As an example, we consider the twist of the Lie algebra $s l(2)$ related to the Toda lattices associated to the $\mathcal{D}_{n}$ root system.

This work was partially supported by RFBR grant. 


\section{References}

[1] L.D. Faddeev. In J B Zuber and R Stora, editors, Les Houches Lectures, pages 719-756, Amsterdam, 1984. North-Holland.

[2] P.P. Kulish and E.K. Sklyanin. Quantum Spectral Transform Method. In J. Hietarinta and C. Montonen, editors, Integrable Quantum Field Theories, Lecture Notes in Physics, volume 151, pages $61-119$, Berlin, 1982. Springer.

[3] I.V. Cherednik. Theor.Math.Phys., 61:977, 1984.

[4] E.K. Sklyanin. J.Phys.A., A21:2375-2389, 1988.

[5] V.B. Kuznetsov and A.V. Tsiganov. Zap.Nauchn.Seminars LOMI, 172:89-98, 1989.

[6] H.J. De Vega and A. Gonzalez-Ruiz. J.Phys., A27:6129-6138, 1993.

[7] P.P. Kulish and R. Sasaki. Prog. of Theor.Phys., 89(3):741-761, 1993.

[8] A.Yu. Alekseev, L.D. Faddeev, and M.A. Semenov-Tian-Shansky. Comm.Math.Phys., 149:335$345,1992$.

[9] A.Yu. Alekseev and L.D. Faddeev. Comm.Math.Phys., 141:413, 1991.

[10] V.G. Drinfeld. Leningrad Math.J., 1:1419-1457, 1990.

[11] O.I. Bogoyavlensky. Commun.Math.Phys., 51(3):201-209, 1976.

[12] S. Khoroshkin, A. Stolin, and V. Tolstoy, in: From Field Theory to Quantum Groups, eds. B. Jancewicz and J. Sobczyk, WS, pp.53-77, 1996.

[13] V.I. Inozemtsev. Commun.Math.Phys., 121:629-643, 1989.

[14] A.V. Tsiganov. Automorphisms of $s l(2)$ and dynamical $r$-matrices. solv-int/9610003, 1996.

[15] V.B. Kuznetsov. J.Phys.A., 30:2127-2138, 1997.

[16] J.-M. Maillet and J. Sanchez de Santos. Drinfeld twists and algebraic Bethe ansatz. 1996. Galg/9612012.

[17] E.K. Sklyanin. Progr.Theor.Phys.Suppl., 118:35-61, 1995.

[18] E.K. Sklyanin. The quantum Toda chain. In Sanchez N, editor, Non-linear equations in classical and quantum field theory. Lecture Notes in Phys. 226, page 196. Springer, 1985.

[19] V.B. Kuznetsov and A.V. Tsiganov. Zap.Nauchn.Seminars POMI, 205:81-89, 1993.

[20] V.B. Kuznetsov and A.V. Tsiganov. J.Phys., A22:L73-L79, 1989. 